\title{
Desempenho fisiológico de sementes e plântulas de três espécies florestais submetidas à salinidade por $\mathrm{KCl}$
}

\author{
Physiological performance of seeds and seedlings of forest species submitted to salinity by $\mathrm{KCl}$ \\ Comportamiento fisiológico de semillas y plántulas de especies forestales sometidas a salinidad por \\ $\mathrm{KCl}$
}

\author{
Andrezza Lorena Queiroz Pamplona \\ ORCID: https://orcid.org/0000-0002-2262-1874 \\ Universidade Federal Rural da Amazônia, Brasil \\ E-mail: andrezzapamplona@yahoo.com.br \\ Evilin Nascimento Costa \\ ORCID: https://orcid.org/0000-0003-4956-9284 \\ Universidade Federal Rural da Amazônia, Brasil \\ E-mail: evilinfloresta@yahoo.com.br \\ Juliane Silva Brasil Carvalho \\ ORCID: https://orcid.org/0000-0002-6672-8980 \\ Universidade Federal Rural da Amazônia, Brasil \\ E-mail: julianebrasilflorestal@hotmail.com \\ Sebastião Rodrigo do Remédio Souza de Oliveira \\ ORCID: https://orcid.org/0000-0002-9498-8237 \\ Universidade Federal Rural da Amazônia, Brasil \\ E-mail: rodrigosouza6789@gmail.com \\ Sara Souza de Jesus de Oliveira \\ ORCID: https://orcid.org/0000-0003-1879-9335 \\ Universidade Federal Rural da Amazônia, Brasil \\ E-mail: sara.jesus0303@gmail.com \\ Carla Topázio Gomes das Chagas \\ ORCID: https://orcid.org/0000-0003-0626-1394 \\ Universidade Federal Rural da Amazônia, Brasil \\ E-mail: carlatoppazio@gmail.com \\ Henry Albert Werner \\ ORCID: https://orcid.org/0000-0002-1373-9766 \\ Universidade Federal Rural da Amazônia, Brasil \\ E-mail: henrywerner.hw@gmail.com \\ Denes de Souza Barros \\ ORCID: https://orcid.org/0000-0001-5317-3923 \\ Universidade Federal Rural da Amazônia, Brasil \\ E-mail: denesbarros@yahoo.com.br \\ Vanessa Mayara Souza Pamplona \\ ORCID: https://orcid.org/0000-0002-2461-2103 \\ Universidade Federal Rural da Amazônia, Brasil \\ E-mail: vanessa.pamplona@ufra.edu.br \\ Bárbara Rodrigues de Quadros \\ ORCID: https://orcid.org/0000-0001-7052-4326 \\ Universidade Federal Rural da Amazônia, Brasil \\ E-mail: barbara.quadros@ufra.edu.br
}

\begin{abstract}
Resumo
O objetivo desta pesquisa foi avaliar o desempenho fisiológico de sementes e plântulas de paricá, (Schizolobium parahyba var. amazonicum (Huber x Ducke) Barneby), paineira (Ceiba speciosa (A.St. Hil ex Brako) Ravenna) e pau de balsa (Ochroma pyramidale (Cav. ex Lam.) Urb.), submetidas a diferentes níveis de salinidade por cloreto de potássio $(\mathrm{KCl})$. O delineamento experimental adotado foi inteiramente casualizado. A avaliação do efeito do estresse salino foi realizada por meio da umidificação das sementes em soluções aquosas de $\mathrm{KCl}$ nas concentrações: 0; 25; 50; 75; $100 \mathrm{mM}$. Foram avaliadas as variáveis: porcentagem de germinação, primeira contagem, índice de velocidade de germinação, comprimento da parte aérea, comprimento da raiz, massa fresca e seca. Foram observadas que as sementes de paricá e paineira-rosa possuíram sua germinação, índice de velocidade de germinação e a primeira contagem, reduzida conforme o aumento das concentrações de $\mathrm{KCl}$. As plântulas apresentaram redução no comprimento da raiz,
\end{abstract}


massa fresca e seca do comprimento total de maneira proporcional ao aumento das dosagens, demonstrando intolerância dessas espécies a altas concentrações de $\mathrm{KCl}$. As sementes e plântulas de pau de balsa com exceção as variáveis massa seca e primeira contagem, apresentaram tolerância as concentrações de $\mathrm{KCl}$ até alcançar os valores máximo próximos a $50 \mathrm{mM}$ da solução tratamento.

Palavras-chave: Desenvolvimento vegetal; Intolerância; Estresse salino.

\begin{abstract}
The objective of this research was to evaluate the physiological development of seeds and seedlings of de paricá, (Schizolobium parahyba var. amazonicum (Huber x Ducke) Barneby), paineira (Ceiba speciosa (A.St. Hil ex Brako) Ravenna) and pau de balsa (Ochroma pyramidale (Cav. ex Lam.) Urb.), submitted to different levels of salinity by potassium chloride $(\mathrm{KCl})$. The experimental design adopted was entirely randomized. The evaluation of the effect of salt stress was done by moistening the seeds in aqueous solutions of $\mathrm{KCl}$ at concentrations: $0 ; 25 ; 50 ; 75 ; 100 \mathrm{mM}$. The following variables were evaluated: germination, first count, germination speed index, aerial part length, root length, fresh and dry mass. It was observed that the seeds of paricá and paineira had their germination, germination speed index and first count, reduced as the concentrations of $\mathrm{KCl}$ increased. The seedlings presented reduction in root length, fresh mass and dry of the total length in a proportional way to the increase of the dosages, demonstrating intolerance of these species to high concentrations of $\mathrm{KCl}$. The seeds and seedlings of pau de balsa with exception to the variables dry mass and first count, showed tolerance to the concentrations of $\mathrm{KCl}$ until reaching the maximum values close to $50 \mathrm{mM}$ of the treatment solution.
\end{abstract}

Keywords: Plant development; Intolerance; Salt stress.

\title{
Resumen
}

El objetivo de esta investigación fue evaluar el desarrollo fisiológico de semillas y plántulas de paricá, (Schizolobium parahyba var. amazonicum (Huber x Ducke) Barneby), paineira (Ceiba speciosa (A.St. Hil ex Brako) Ravenna) y palo de balsa (Ochroma pyramidale (Cav. ex Lam.) Urb.), sometidas a diferentes niveles de salinidad por cloruro de potasio $(\mathrm{KCl})$. El diseño experimental adoptado fue completamente al azar. La evaluación del efecto del estrés salino se realizó humedeciendo las semillas en soluciones acuosas de $\mathrm{KCl}$ en las concentraciones: $0 ; 25 ; 50 ; 75 ; 100$ mm. Se evaluaron las variables: germinación, primer conteo, índice de velocidad de germinación, longitud de brote, longitud de raíz, masa fresca y seca. Se observó que las semillas de paricá y paineira tuvieron su germinación, índice de velocidad de germinación y primer conteo, reducidos a medida que aumentaron las concentraciones de $\mathrm{KCl}$. Las plántulas mostraron una reducción en el largo de la raíz, masa fresca y seca del largo total que es proporcional al aumento de dosis, demostrando intolerancia de estas especies a altas concentraciones de $\mathrm{KCl}$. Las semillas y plántulas de palo de balsa, con excepción de las variables masa seca y primer recuento, mostraron tolerancia a las concentraciones de $\mathrm{KCl}$ hasta alcanzar los valores máximos cercanos a los $50 \mathrm{~mm}$ de la solución de tratamiento.

Palabras clave: Desarrollo vegetal; Intolerância; Estrés salino.

\section{Introdução}

Muitas tecnologias foram fundamentais na expansão das fronteiras agrícolas, como a utilização da irrigação por exemplo, que possibilitou a utilização de áreas no qual não seria possível, devido à deficiência hídrica do solo de algumas regiões (Souza, 2013).

A deficiência hídrica pode provocar alteração das propriedades químicas do solo, elevando principalmente as concentrações salinas. Como consequência, apresenta alterações na fertilidade do solo, sendo prejudiciais a microbiota que exercem atividade no solo, iniciando assim o processo de degradação (Bezerra et al., 2020; Castro, Santos, Araújo, 2019).

Além disso, a adubação excessiva através de fontes orgânicas de origem animal (dejeto de suíno, cama de frango), industriais (lodo de curtume) e urbanos (lodo de esgoto), tornaram-se comuns nos campos de agricultura no Brasil. No entanto esses resíduos possuem em sua constituição elementos chamados de metais pesados que são prejudiciais ao solo e também aos vegetais não tolerantes (Matoso, Salman, 2016)

A salinização, além de problemas as propriedades do solo, também afetam a germinação de sementes de espécies vegetais, além de serem causadores de muitos outros problemas de ordem fisiológica (redução do potencial osmótico e limitação da absorção de água) metabólicas e anatômicas (Bezerra et al., 2020; Dutra et al., 2017).

Neste contexto, visando reduzir os impactos causados as propriedades do solo, torna-se necessário o emprego de medidas mitigadoras, no entanto, dependendo do nível de salinidade presente no solo, alguns métodos são bastantes dificultosos e pouco eficientes (Mendonça, 2006). Assim, surgiram algumas alternativas, como por exemplo o plantio de 
espécies leguminosas com propriedades capazes de promover a extração de elementos químicos do solo causadores das salinidades, servindo também como fonte de adubação (adubo verde). Além disso, esses vegetais também servem como fonte de cobertura do solo, e consequentemente reduzem a possibilidade de erosão, melhorando assim a estrutura do solo (Mishra $e t$ al., 2003, Pedrotti et al., 2015, Su et al., 2005).

Então, o conhecimento das propriedades dos vegetais é imprescindível quando se pretende utiliza-la na recuperação de áreas degradadas (Guedes et al., 2011). Além disso, há de se considerar também a importância das espécies florestais que possam serem destinadas a atender a demanda industrial, agregando valor a matéria prima. Seguindo essa temática, o desenvolvimento de estudos e pesquisas que permitam definir o nível de tolerância de uma espécie às limitações do ambiente, bem como obter informações que possam subsidiar plantios em áreas sobre elevado níveis de salinidade são fundamentais (Lavezo et al., 2015).

Estudos que envolvam a determinação de níveis salinos não deletérios ao processo germinativo de sementes de espécies florestais ganham relevância, pois tendem a possibilitar maior habilidade dessas espécies a tolerar as diferentes condições ambientais de estresse, inclusive salino, lhes permitindo a habilidade de sobrevivência e assegurando a perpetuação dessas espécies em diferentes ambientes (Lemes, 2011).

Algumas pesquisas simulando o efeito do estresse salino na germinação e no vigor de sementes de espécies florestais têm sido desenvolvidas, a exemplo daquelas com sementes de Enterolobium schomburgkii Benth., (Braga et al., 2009), Gliricidia sepium (Jacq.) Steud., (Farias et al., 2009) e Zizyphus joazeiro Mart., (Lima e Torres, 2009), com o intuito de saber como estas espécies florestais se comportam em meio a esse tipo de estresse. De acordo com Souza (2013), distinguir como as espécies vegetais se comportam a diferentes níveis de salinidade nas distintas fases de desenvolvimento é decisivo para garantir o sucesso dos plantios em áreas salinizadas.

Com base nisso, a presente pesquisa tem como objetivo avaliar o desempenho fisiológico de sementes e plântulas de paricá (Schizolobium parahyba var. amazonicum (Huber x Ducke) Barneby), paineira (Ceiba speciosa (A.St. Hil ex Brako) Ravenna) e pau de balsa (Ochroma pyramidale (Cav. ex Lam.) Urb.), submetidas a diferentes níveis de salinidade por cloreto de potássio $(\mathrm{KCl})$.

\section{Metodologia}

O experimento foi conduzido no Laboratório multidisciplinar da Universidade Federal Rural da Amazônia, campus Paragominas. As espécies utilizadas foram das espécies paineira-rosa e pau de balsa, sendo as sementes coletadas no município de Paragominas/Pa. Além de sementes de paricá, coletadas no município de Ouro Preto do Oeste, situado no estado de Rondônia.

As sementes de paricá e paineira foram armazenadas em recipientes de plástico transparente de polietileno com temperatura controladas a $26{ }^{\circ} \mathrm{C}$ por um período de armazenamento até a montagem do teste (quatro e cinco dias, respectivamente). Já as sementes de pau de balsa, forma embaladas em sacos de papel tipo Kraft e acondicionadas em geladeira com temperatura média de $15^{\circ} \mathrm{C}$, por um período de dez dias até a montagem do teste.

Como as sementes das espécies utilizadas nesse trabalho possuem tegumento duro e impermeável à entrada de água, antes dos tratamentos, passaram pelo processo de superação de dormência, buscando uniformizar e acelerar o processo germinativo. A superação de dormência das sementes de paineira-rosa, foram tratadas através da escarificação química em solução de ácido sulfúrico $\left(\mathrm{H}_{2} \mathrm{SO}_{4}\right)$ concentrado por um período de 20 minutos e em seguida lavadas abundantemente em água corrente (Nascimento, 2012). Para o paricá, as sementes foram escarificadas manualmente, atingindo o tegumento com uma lixa, na lateral do terço superior da semente, parte oposta a micrófila, sem atingir os cotilédones. O procedimento utilizado no pau de balsa, consistiu na imersão das sementes em água aquecida até a temperatura de $80{ }^{\circ} \mathrm{C}$, deixando as na mesma na água 
até atingir a temperatura ambiente; metodologia usada de acordo com as instruções para análise de sementes de espécies florestais (Brasil, 2013).

Para avaliar o efeito do estresse salino, as sementes foram umedecidas com soluções aquosas de cloreto de potássio (KCl) nas concentrações: 0 (controle com água destilada); 25; 50; 75; $100 \mu \mathrm{M}$.

Foram determinadas as variáveis: porcentagem de germinação; primeira contagem (PC), índice de velocidade de germinação (IVG), Comprimento da parte aérea (CPA), comprimento da raiz principal (CR), massa fresca total (MF) e massa seca total (MS).

Para a germinação do paricá e da paineira, as sementes foram distribuídas sobre papel tipo germiteste, que posteriormente foram umedecidos na proporção de 3,0 vezes o peso do papel. Os rolos do papel foram acondicionados em sacos plásticos transparentes, de $0,04 \mathrm{~mm}$ de espessura. Na germinação da paineira e do paricá, o teste teve duração de 10 dias, com temperatura constante de $25^{\circ} \mathrm{C}$, fotoperíodo de 12 horas e umidade relativa $90 \%$.

Para a germinação do pau de balsa, as sementes foram semeadas em papel tipo mata-borrão, que foram acondicionados em caixas plásticas transparentes (Gerbox). O teste de germinação seguiu as instruções para análise de sementes de espécies florestais (Brasil, 2013). A duração do teste com pau de balsa foi de 21 dias, com temperatura constante de $35{ }^{\circ} \mathrm{C}$, fotoperíodo de 12 horas e umidade relativa $90 \%$.

Foram computadas como germinadas as sementes com protusão da radícula com dimensão $\geq 2 \mathrm{~mm}$ segundo metodologia proposta por Lemes et al., (2012). A primeira contagem das sementes foi realizada conforme Brasil (2013), considerando como teste de vigor as sementes que germinam mais rapidamente, com maior porcentagem de plântulas normais nessa data, sendo consideradas como mais vigorosas. A primeira contagem de germinação (PC) das sementes de paricá e paineira foram realizadas no $7^{\circ}$ dia após a semeadura e do pau de balsa ocorreu no $13^{\circ}$ dia após a semeadura. Já o índice de velocidade de germinação (IVG) foram realizados juntamente com teste de germinação, com valores de sementes germinadas dia a dia, através da somatória do número de sementes germinadas em cada dia (não cumulativo), dividida pelo número de dias decorridos entre a semeadura e a germinação (Maguire, 1962).

Após a contagem final do teste de germinação, foram retiradas aleatoriamente cinco amostras de plântulas normais de cada tratamento e repetição para medições de comprimento da parte aérea e da raiz principal, com auxílio de uma régua graduada em centímetros, sendo os resultados expressos em cm/plântula. Utilizando as mesmas amostras de cinco plântulas, também foram quantificadas a massa fresca e a massa seca de plântulas. Para a massa fresca, foi feita a pesagem das plântulas normais com balança analítica de 0,001 g de precisão. Para a realização da massa seca, as plântulas da massa fresca foram acondicionadas em sacos de papel Kraft, identificadas e levadas à estufa de ventilação forçada, regulada a $80^{\circ} \mathrm{C}$ por 24 h, após, estas, foram pesadas e feita a média por repetição para ambas as variáveis (Nakagawa, 1999).

Para a análise estatística, os dados obtidos foram submetidos ao teste de Shapiro-Wilk para testar normalidade da distribuição, posteriormente aplicou-se a análise de variância. Para descrever a variação dos parâmetros avaliados em função das doses de $\mathrm{KCl}$ foram ajustadas equações de regressão polinomial, sendo escolhido o modelo de maior grau e com maior valor de $\mathrm{R}^{2}$. Para a realização dos testes utilizou-se o programa AgroEstat (2015). A estimação das equações de regressão foi realizada com o auxílio do programa Excel, utilizado o nível de significância igual a 5\%.

\section{Resultados}

Nesse sentido, foi observado que houve efeito significativo das doses de $\mathrm{KCl}$ na variável percentual de germinação e índice de velocidade de germinação para todas as espécies avaliadas neste estudo. No percentual de germinação das sementes de paricá e paineira o efeito foi de primeira ordem, com comportamento decrescente, ou seja, a germinação era reduzida na proporção de $0,39 \%$ na medida em que houve o aumento das concentrações da solução de $\mathrm{KCl}$. Já para a espécie pau de balsa, 
foi observado comportamento quadrático, com a germinação máxima obtida quando a dosagem foi igual a 50,71 $\mu \mathrm{M}$ da concentração de $\mathrm{KCl}$ (Tabela 1; Figura 1A).

Tabela 1. Resumo da análise de variância para as variáveis (G) percentual de germinação e (IVG) índice de velocidade de germinação para as sementes de paricá, paineira e pau de balsa, submetidas a diferentes níveis de salinidade por cloreto de potássio, com partição dos efeitos em regressões polinomiais.

\begin{tabular}{|c|c|c|c|c|c|c|c|}
\hline \multirow{2}{*}{ Causas de Variação } & \multirow{2}{*}{ GL } & \multicolumn{3}{|c|}{ G $(\%)$} & \multicolumn{3}{|c|}{ IVG } \\
\hline & & Paricá & Paineira & Pau de balsa & Paricá & Paineira & Pau de balsa \\
\hline Doses de $\mathrm{KCl}$ & 4 & $8,81^{* *}$ & $5,05^{* *}$ & $17,01 * *$ & $70,82^{* *}$ & $40,27 * *$ & $34,30 * *$ \\
\hline Resíduos & 15 & - & - & - & - & - & - \\
\hline Regressão Linear & 1 & $30,08 * *$ & $18,86^{* *}$ & $0,20^{\mathrm{NS}}$ & $173,98^{* * *}$ & $40,27 * *$ & $0,00^{\mathrm{NS}}$ \\
\hline Regressão Quadrática & 1 & $2,59^{\mathrm{NS}}$ & $0,45^{\mathrm{NS}}$ & $65,53 * *$ & $0,02^{\mathrm{NS}}$ & $7,99 *$ & $133,57 * *$ \\
\hline Regressão Cúbica & 1 & $0,61^{\mathrm{NS}}$ & $0,07^{\mathrm{NS}}$ & $0,12^{\mathrm{NS}}$ & $50,43 * *$ & $0,06^{\mathrm{NS}}$ & $0,80^{\mathrm{NS}}$ \\
\hline
\end{tabular}

* - significativo a $5 \%$ de probabilidade; ${ }^{\text {NS }}$ - Não significativo a $5 \%$ de probabilidade.

Fonte: Autores (2021).

Figura 1. Médias observadas para as variáveis percentual de germinação (G) e índice de velocidade de germinação (IVG) para as sementes de paricá, paineira e pau de balsa, submetidas a diferentes concentrações de soluções aquosas de cloreto de potássio $(\mathrm{KCl})$.

A

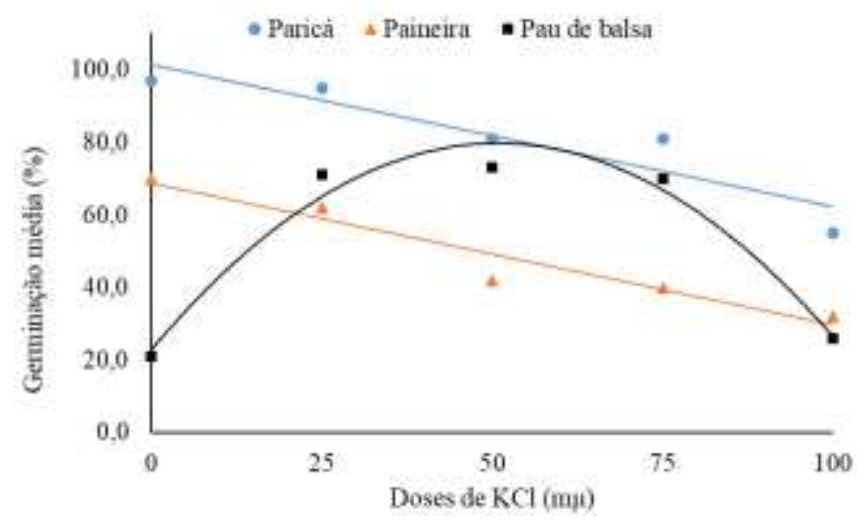

G paricá $=-101,40+0,39 x\left(R^{2}=0,85\right)$

G paineira $=68,80-0,39 x\left(R^{2}=0,93\right)$

$\mathrm{G}$ pau de balsa $=22,83+2,24 \mathrm{x}-0,02 \mathrm{x}^{2}\left(\mathrm{R}^{2}=0,97\right)$

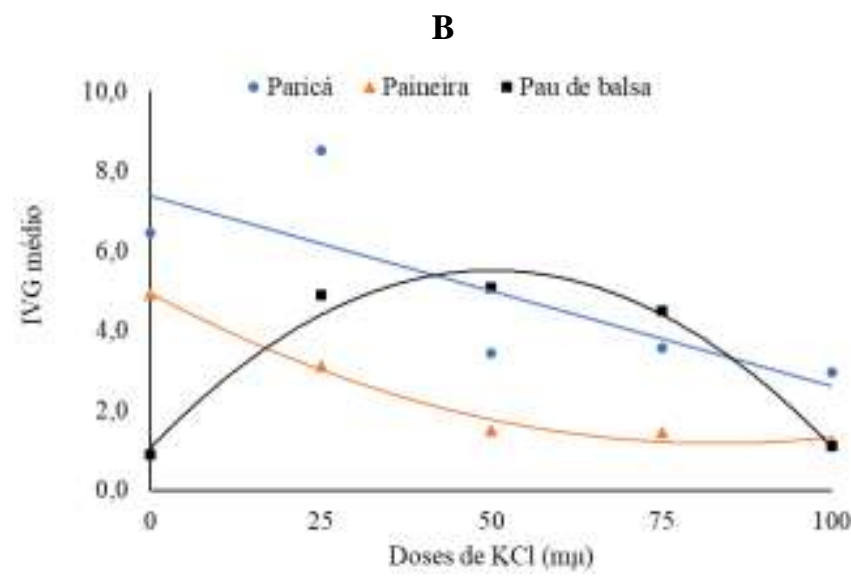

IVG paricá $=7,39-0,05 x\left(R^{2}=0,61\right)$

IVG paineira $=4,94-0,09 \mathrm{x}+0,0005 \mathrm{x}^{2}\left(\mathrm{R}^{2}=0,99\right)$

IVG p. balsa $=1,06+0,18 \mathrm{x}-0,002 \mathrm{x}^{2}\left(\mathrm{R}^{2}=0,97\right)$

Fonte: Autores (2021).

Para o índice de velocidade de germinação, foi notado comportamento linear (decrescente) apenas para as sementes de paricá, com decréscimo de 0,05 por cada unidade de $\mathrm{KCl}$ acrescida. As sementes de paineiras e pau de balsa apresentaram comportamento quadrático, no entanto, foi notado que para a paineira a velocidade mínima de germinação foi obtida quando a concentração da solução de $\mathrm{KCl}$ foi igual a $90,5 \mu \mathrm{M}$, já o pau de balsa, a velocidade de germinação máxima foi observada quando as dosagens da solução tratamento alcançou 49,44 $\mu \mathrm{M}$ (Tabela 1; Figura 1B).

Para a variável comprimento da parte aérea, houve diferença significativa apenas para as plântulas de paricá e pau de balsa, apresentando comportamento quadrático para ambas. Para as plântulas de paricá, o comprimento da mínimo da parte aérea ocorreu no momento que a dosagem atingiu 116,16 $\mu \mathrm{M}$ da concentração de $\mathrm{KCl}$. Para o pau de balsa o comprimento máximo foi observado quando a dosagem do tratamento foi de $48 \mu \mathrm{M}$ da concentração de $\mathrm{KCl}$. Em relação as plântulas de paineira, não foi notado efeito significativo das doses para essa variável (Tabela 2; Figura 2A). 
Tabela 2. Resumo da análise de variância para as variáveis comprimento da parte aérea (CPA) e comprimento da raiz (CR) para as sementes de paricá, paineira e pau de balsa, submetidas a diferentes níveis de salinidade por cloreto de potássio, com partição dos efeitos em regressões polinomiais.

\begin{tabular}{|c|c|c|c|c|c|c|c|}
\hline \multirow{2}{*}{ Causas de Variação } & \multirow{2}{*}{ GL } & \multicolumn{3}{|c|}{ CPA (cm) } & \multicolumn{3}{|c|}{ CR $(\mathbf{c m})$} \\
\hline & & Paricá & Paineira & Pau de balsa & Paricá & Paineira & Pau de balsa \\
\hline Doses de $\mathrm{KCl}$ & 4 & $53,16^{* *}$ & $1,92^{\mathrm{NS}}$ & $5,67 * *$ & $11,22 * *$ & $3,61 * *$ & $2,14^{\mathrm{NS}}$ \\
\hline Resíduos & 15 & - & - & - & - & - & - \\
\hline Regressão Linear & 1 & $139,90 * *$ & $4,51^{\mathrm{NS}}$ & $0,08^{\mathrm{NS}}$ & $40,04 * *$ & $9,82 * *$ & $0,07^{\mathrm{NS}}$ \\
\hline Regressão Quadrática & 1 & $10,32 * *$ & $2,21^{\mathrm{NS}}$ & $20,67 * *$ & $0,04^{\mathrm{NS}}$ & $2,12^{\mathrm{NS}}$ & $8,15^{*}$ \\
\hline Regressão Cúbica & 1 & $32,74 * *$ & $0,83^{\mathrm{NS}}$ & $0,10^{\mathrm{NS}}$ & $0,26^{\mathrm{NS}}$ & $0,74^{\mathrm{NS}}$ & $0,31^{\mathrm{NS}}$ \\
\hline
\end{tabular}

* - significativo a $5 \%$ de probabilidade; ${ }^{\text {NS }}$ - Não significativo a $5 \%$ de probabilidade.

Fonte: Autores (2021).

Figura 2. Médias observadas para as variáveis comprimento da parte aérea (CPA) e comprimento da raiz (CR) para as sementes de paricá, paineira e pau de balsa, submetidas a diferentes concentrações de soluções aquosas de cloreto de potássio $(\mathrm{KCl})$.

A

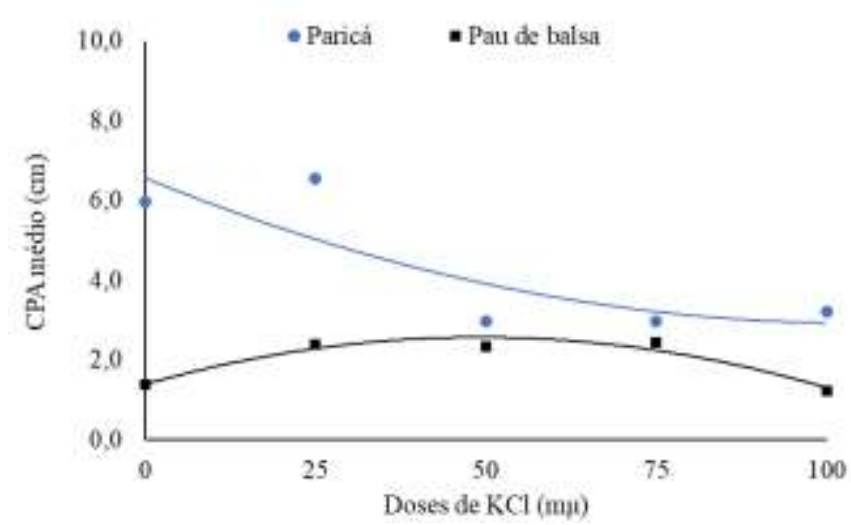

CPA paricá $=6,56-0,07 x+0,0003 x^{2}\left(R^{2}=0,71\right)$

CPA p. balsa $=1,39+0,05 x-0,0005 x^{2}\left(R^{2}=0,91\right)$

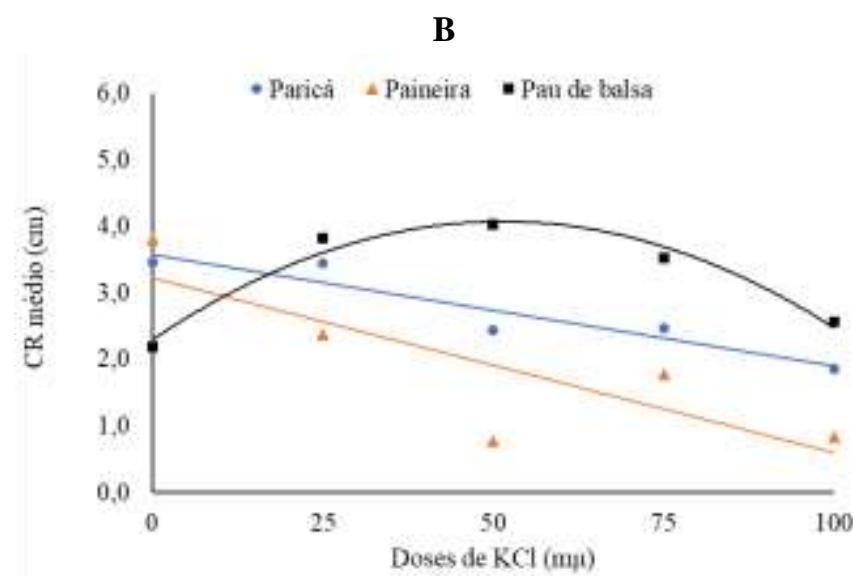

CR paricá $=3,58-0,017 \mathrm{x}\left(\mathrm{R}^{2}=0,89\right)$

CR paineira $=3,22-0,03 x\left(R^{2}=0,68\right)$

CR p. balsa $=2,29+0,07 x-0,0007 x^{2}\left(R^{2}=0,96\right)$

Fonte: Autores (2021).

Para o crescimento da raiz, houve efeito significativo para as três espécies estudadas, com comportamento linear negativo para as plântulas de paricá e paineira, com redução de 0,017 e 0,03 cm respectivamente, no tamanho da raiz. Para as plântulas de pau de balsa o efeito de regressão foi quadrático, como o comprimento máximo da raiz observado quando a dosagem de $\mathrm{KCl}$ foi igual a $50 \mu \mathrm{M}$ (Tabela 2; Figura 2B).

A massa fresca para as três espécies apresentou diferença significativa, sendo que houve tendência linear negativa para as plântulas de paricá, com o decréscimo de $0,02 \mathrm{~g}$ por cada unidade de solução tratamento acrescida. Já as plântulas de paineira e pau de balsa apresentaram comportamento quadrático, sendo que a massa fresca mínima da paineira foi observada quando a dosagem de $\mathrm{KCl}$ foi igual a $53,7 \mu \mathrm{M}$.

Em relação ao pau de balsa foi notado que a massa seca máxima foi alcançada quando a dosagem de $\mathrm{KCl}$ foi igual a 48,92 $\mu \mathrm{M}$ (Tabela 3; Figura 3A). Para a massa seca não houve regressão para as plântulas de paricá e pau de balsa, ocorrendo efeito para essa variável apenas para as plântulas de paineira, apresentando comportamento de segunda ordem, sendo que a massa seca mínima foi obtida quando a dosagem de $\mathrm{KCl}$ foi igual a 49,25 $\mu \mathrm{M}$ (Tabela 3; Figura 3B). 
Tabela 3. Resumo da análise de variância para as variáveis massa fresca (MF) e massa seca (MS) para as plântulas de paricá, paineira e pau de balsa, submetidas a diferentes níveis de salinidade por cloreto de potássio, com partição dos efeitos em regressões polinomiais.

\begin{tabular}{|c|c|c|c|c|c|c|c|}
\hline \multirow{2}{*}{ Causas de Variação } & \multirow{2}{*}{ GL } & \multicolumn{3}{|c|}{ MF (g) } & \multicolumn{3}{|c|}{ MS (g) } \\
\hline & & Paricá & Paineira & Pan de balsa & Paricá & Paineira & Pau de balsa \\
\hline Doses de $\mathrm{KCl}$ & 4 & $5,92^{* *}$ & $4,89^{\circ}$ & $17,14^{* *}$ & $1,63^{\mathrm{NS}}$ & $8,11^{* *}$ & $0,76^{\mathrm{NS}}$ \\
\hline Residuos & 15 & - & - & $\cdot$ & $=$ & - & - \\
\hline Regressão Linear & 1 & $20,81^{* * *}$ & $1,52^{\mathrm{NS}}$ & $4,32^{\mathrm{NS}}$ & $2,81^{\mathrm{N5}}$ & $4,67^{*}$ & $1,31^{\mathrm{NS}}$ \\
\hline Regressăo Quadrática & 1 & $1,70^{\mathrm{Ns}}$ & $10,13^{* *}$ & $56,08 * *$ & $1,31^{\mathrm{NS}}$ & $15,95^{* *}$ & $0,14^{\mathrm{Ns}}$ \\
\hline Regressão Cúbica & 1 & $0,62^{\mathrm{Ns}}$ & $1,90^{\mathrm{NS}}$ & $0,59^{\mathrm{NS}}$ & $0,64^{\mathrm{NS}}$ & $9,93^{\circ *}$ & $1,42^{\mathrm{NS}}$ \\
\hline
\end{tabular}

* significativo a $5 \%$ de probabilidade; ${ }^{\text {NS }}$ - Não significativo a $5 \%$ de probabilidade.

Fonte: Autores (2021).

Figura 3. Médias observadas para as variáveis massa fresca (MF) e massa seca (MS) para as plântulas de paricá, paineira e pau de balsa, submetidas a diferentes concentrações de soluções aquosas de cloreto de potássio $(\mathrm{KCl})$.

A

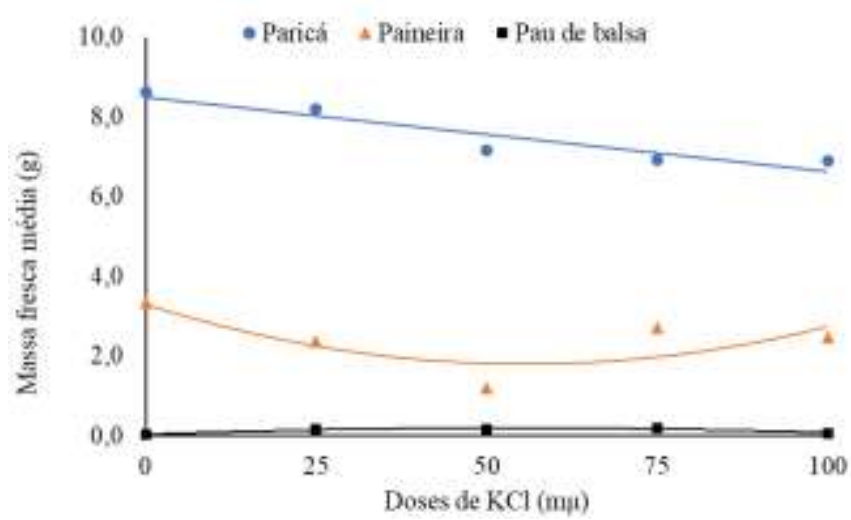

MF paricá $=8,50-0,02 x\left(\mathrm{R}^{2}=0,88\right)$

MF paineira $=3,29-0,05 x+0,0005 \mathrm{x}^{2}\left(\mathrm{R}^{2}=0,59\right)$

MF p. balsa $=0,03+0,01 \mathrm{x}-0,00006 \mathrm{x}^{2}\left(\mathrm{R}^{2}=0,88\right)$
B

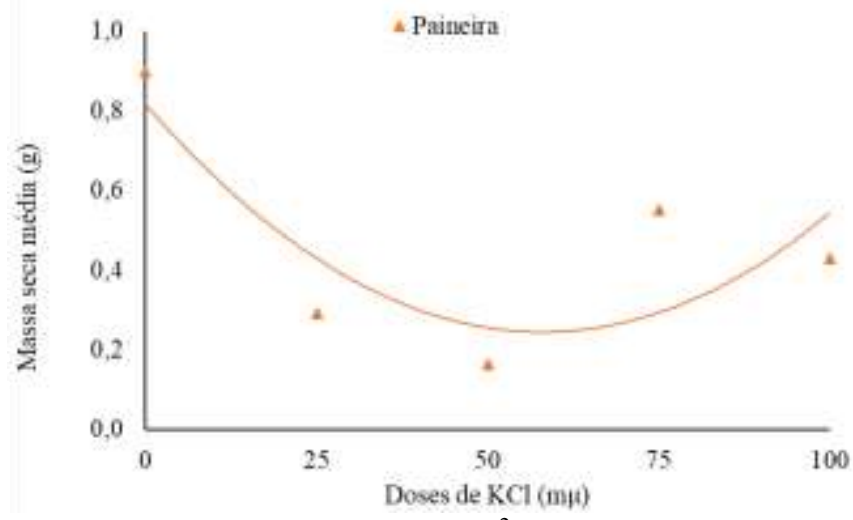

MS paineira $=0,81-0,08 x+0,0002 x^{2}\left(R^{2}=0,64\right)$

Fonte: Autores (2021).

Para a variável comprimento total, as plântulas de paricá apresentaram tendência linear negativa, com redução de 0,052 cm por cada unidade de solução tratamento adicionada. As plântulas de paricá e pau de balsa demonstraram tendência quadrática, sendo que no paricá o comprimento total mínimo foi observado quando a solução de $\mathrm{KCl}$ foi igual a 140,83 $\mu \mathrm{M}$. E o pau de balsa o comprimento total máximo foi obtido quando a dosagem do tratamento foi igual a $48,92 \mu \mathrm{M}$ de $\mathrm{KCl}$ (Tabela 4; Figura 4A).

Em relação a primeira contagem, as plântulas de paricá e paineira apresentaram comportamento linear decrescente, com redução de 0,46 e 0,42 respectivamente, para cada unidade de $\mathrm{KCl}$ acrescida na solução. As plântulas de pau de balsa não apresentaram efeito significativo para essa variável (Tabela 4; Figura 4B). 
Tabela 4. Resumo da análise de variância para comprimento total (CT) e primeira contagem (PC) para as plântulas de paricá, paineira e pau de balsa, submetidas a diferentes níveis de salinidade por cloreto de potássio, com partição dos efeitos em regressões polinomiais.

\begin{tabular}{|c|c|c|c|c|c|c|c|}
\hline \multirow{2}{*}{ Causas de Variação } & \multirow{2}{*}{ GL } & \multicolumn{3}{|c|}{$\mathrm{CT}(\mathrm{cm})$} & \multicolumn{3}{|c|}{ PC } \\
\hline & & Paricá & Paineira & Pau de balsa & Paricá & Paineira & Pau de balsa \\
\hline Doses de $\mathrm{KCl}$ & 4 & $59,54 * *$ & $2,58^{\mathrm{NS}}$ & $3,59 *$ & $10,95 * *$ & 9,06 & $0,89^{\mathrm{NS}}$ \\
\hline Resíduos & 15 & - & - & - & - & - & - \\
\hline Regressão Linear & 1 & $178,28 * *$ & $6,57 *$ & $0,01^{\mathrm{NS}}$ & $33,17 * *$ & $27,32 * *$ & $0,07^{\mathrm{NS}}$ \\
\hline Regressão Quadrática & 1 & $5,46 *$ & $2,35^{\mathrm{NS}}$ & $13,93 * *$ & $0,30^{\mathrm{NS}}$ & $3,06^{\mathrm{NS}}$ & $0,05^{\mathrm{NS}}$ \\
\hline Regressão Cúbica & 1 & $22,71 * *$ & $0,81^{\mathrm{NS}}$ & $0,10^{\mathrm{NS}}$ & $0,56^{\mathrm{NS}}$ & $0,35^{\mathrm{NS}}$ & $0,27^{\mathrm{NS}}$ \\
\hline
\end{tabular}

* - significativo a $5 \%$ de probabilidade; ${ }^{N S}$ - Não significativo a $5 \%$ de probabilidade.

Fonte: Autores (2021).

Figura 4. Médias observadas para as variáveis comprimento total (CT) e primeira contagem (PC) para as plântulas de paricá, paineira e pau de balsa, submetidas a diferentes concentrações de soluções aquosas de cloreto de potássio $(\mathrm{KCl})$.

A

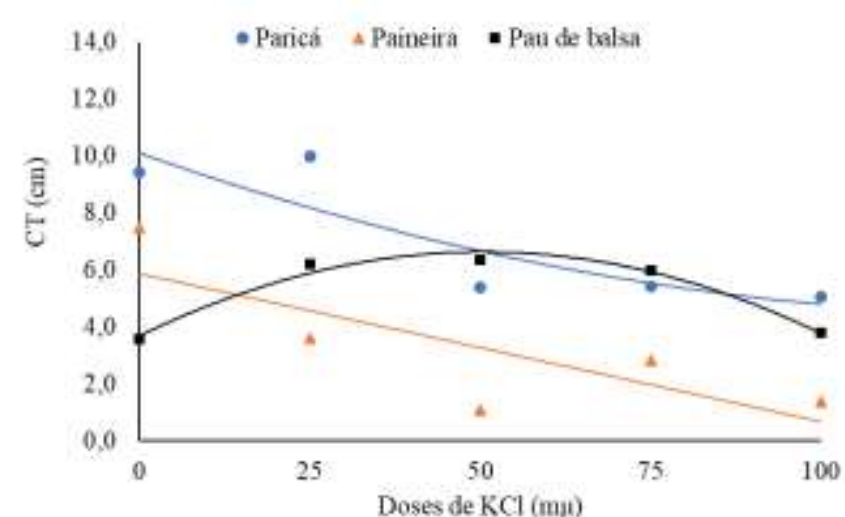

CT paricá $=10,11-0,08 x+0,0003 x^{2}\left(R^{2}=0,77\right)$

CT paineira $=5,88-0,052 x\left(\mathrm{R}^{2}=0,64\right)$

CT p. balsa $=3,68+0,12 \mathrm{x}-0,0012 \mathrm{x}^{2}\left(\mathrm{R}^{2}=0,97\right)$
$\mathbf{B}$

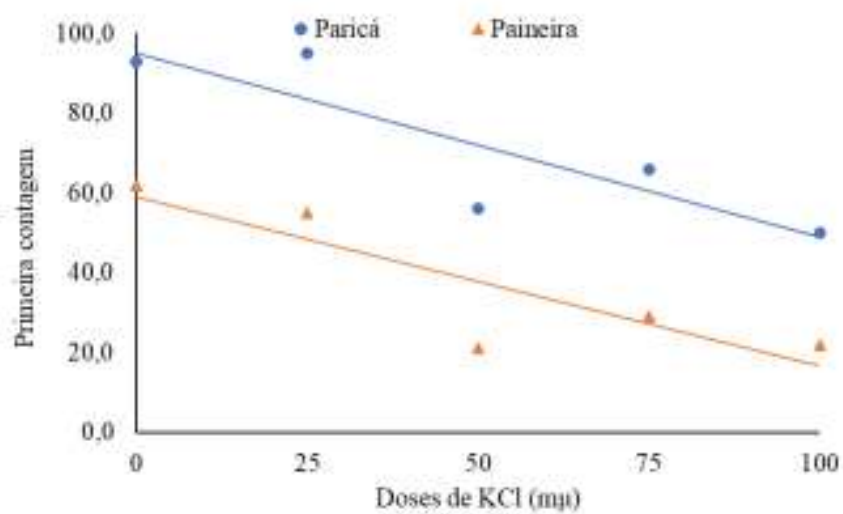

PC paricá $=95-0,46 x\left(R^{2}=0,76\right)$

$\mathrm{PC}$ paineira $=59-0,42 \mathrm{x}\left(\mathrm{R}^{2}=0,75\right)$

Fonte: Autores (2021).

\section{Discussão}

Resultados semelhantes a este estudo foram observados por Silva (2015), trabalhando com sementes de faveira (Parkia platycephala Benth.); Ferreira et al., (2013) estudando o comportamento das sementes de cedro (Cedrela odorata L.) e Silva (2011) analisando as sementes de jurema branca (Piptadenia stipulacea (Benth) Ducke), ambos os autores observaram que o estresse salino, provocado por diferentes concentrações de $\mathrm{KCl}$ promoviam a redução da germinação e do índice de velocidade de germinação. Esse fenômeno pode estar relacionado as altas concentrações de íons presentes na solução, que promovem o estresse fisiológico nas plantas, e consequentemente reduzem o potencial osmótico do meio, retendo água na solução, de forma que o aumento da concentração a torna cada vez menos disponível para as plantas, impedindo a germinação das sementes (Ribeiro et al., 2001; Santos et al., 2016). De acordo com Campos e Assunção (1990), altas concentrações de sais podem ocasionar efeitos osmóticos e iônicos capazes de afetam não apenas a porcentagem de germinação, mas também o índice de velocidade de germinação (IVG).

Em relação a comprimento da parte aérea, Pinto (2013), trabalhando com plântulas de acácia (Acacia mangium Will) observou que o aumento as concentrações de $\mathrm{KCl}$ ocasionou alteração no comprimento da parte aérea. Esse fenômeno pode ser 
explicado pelo estresse causado pelas altas concentrações de sais no metabolismo vegetal, capazes de afetar o crescimento das plantas (Nascimento, 2012). Os efeitos de altas concentrações salinas podem estar diretamente ligadas a alterações de ordem metabólica e fisiológicas, capazes de induzirem a redução da germinação, assim como o índice de velocidade de germinação, provocando também o menor crescimento das plântulas (Santos et al., 2016; Stefanello, Viana, Neves, 2018).

Cruz et al., (2020), estudando o efeito da salinidade na germinação e desenvolvimento de plântulas de pau de balsa, observou que a massa seca, altura e comprimento da raiz das plântulas sofreram influência direta dos teores salinos, devido as mudanças fisiológicas provocas pelos íons presentes na solução.

Diante destes aspectos, observa-se de modo geral a redução no crescimento da maioria das espécies, demostrando provável sensibilidade a salinidade na fase inicial de desenvolvimento. Além disso, há de se destacar que o excesso de sais faz com que o potencial hídrico do ambiente radicular diminua e dificulte a absorção de água do solo (Costa et al., 2003). Assim, os processos de divisão e alongamento celular são afetados e o desenvolvimento do sistema radicular das plantas é reduzido sob condições de déficit hídrico (Mayer; Poljakoff-Mayber, 1989).

Estudos sugerem que sob influência de salinidade do $\mathrm{KCl}$, ocorre uma redução da tolerância das sementes e plântulas das espécies florestais nativas estudadas, verificada pela menor capacidade de transformação do suprimento de reservas dos tecidos de armazenamento ou menor incorporação destes pelo eixo embrionário (Stefanello et al., 2006).

\section{Conclusão}

As sementes de paricá e paineira possuíram sua germinação, índice de velocidade de germinação e primeira contagem reduzida conforme houve aumento das concentrações salinas de $\mathrm{KCl}$.

As plântulas apresentaram redução no comprimento da raiz principal, massa fresca e massa seca do comprimento total de maneira proporcional ao aumento das dosagens, isso demostra intolerância dessas espécies a altas concentrações de $\mathrm{KCl}$.

As sementes e plântulas de pau de balsa com exceção as variáveis massa seca e primeira contagem, apresentaram tolerância as diferentes concentrações de $\mathrm{KCl}$ até alcançar os valores máximo próximas a $50 \mathrm{mM}$ da solução tratamento.

\section{Referências}

Bezerra, L. T., Santos, A. R. C. S., Farias, A. S., Couto, P. C., Ferreira, V. M., Araújo Neto, J. C., e Nascimento, H. H. C. (2020). Emergência e crescimento inicial de plântulas de Libidibia ferrea (Mart., ex Tul.) em diferentes níveis de salinidade na água de irrigação. Brazilian Journal of Animal and Environmental Research, 3(3), 1126-1140. DOI: 10.34188/bjaerv3n3-032

Braga, L. F., Sousa, M. P., e Almeida, T. A. (2009). Germinação de sementes de Enterolobium schomburgkii (Benth.) Benth. Submetidas a estresse salino e aplicação de poliamina. Rev. Bras. Pl. Med., Botucatu, 11(1), 63-70.

Brasil. (2013). Ministério da Agricultura, Pecuária e Abastecimento: Instruções para análise de sementes de espécies florestais. Secretaria de Defesa Agropecuária. Brasília, DF: Mapa/ACS, 98p.

Campos, I. S., e Assunção, M. V. (1990). Efeito do cloreto de sódio na germinação e vigor de plântulas de arroz. Revista Pesquisa agropecuária Brasileira, $25(6), 837-843$

Castro, F. C., Santos, A. M., e Araújo, J. F. (2019) A salinização dos solos na concepção da comunidade quilombola de Cupira - Santa Maria da Boa Vista Pernambuco. Ateliê Geográfico, Goiânia-GO, 13(3), 229-243.

Costa, P. H. A., Silva, J. V., Bezerra, M. A., Enéas-Filho, J., Prisco, J. T., e Gomes-Filho, E. (2003). Crescimento e níveis de solutos orgânicos e inorgânicos em cultivares de Vigna unguiculata submetidos à salinidade. Revista Brasileira de Botânica, São Paulo, 26(3), 289-297.

Cruz, V. S., Yamashita, O. M., Karsburg, I. V., Carvalho, M. A. C., Dallacort, R., Rossi, A. P. B., e Silva, I. V. (2020). Efeito da salinidade na germinação e desenvolvimento de plântulas de Ochroma pyramidale. Nativa, Sinop, 8(2), 239-245. DOI: http://dx.doi.org/10.31413/nativa.v8i2.8266

Dutra, T. R., Massad, M. D., Moreira, P. R., e Ribeiro, E. S. M. (2017). Efeito da salinidade na germinação e crescimento inicial de plântulas de três espécies arbóreas florestais. Pesq. flor. bras., Colombo, 37(91), 323-330. 10.4336/2017.pfb.37.91.1447

Farias, S. G. G., Freire, A. L. O., Santos, D. R., Bakke, I. A., e Silva, R. B. (2009). Efeitos dos estresses hídrico e salino na germinação de sementes de gliricidia [Gliricidia sepium (JACQ.) STEUD.]. Revista Caatinga, 22(4), 152-157. 
Ferreira, E. G. B. S., Matos, V. P., Sena, L. H. M. G., Oliveira, R., e Sales, A. G. F. A. (2013). Processo Germinativo e Vigor de Sementes de Cedrela odorata L. sob Estresse Salino. Ciência Florestal, Santa Maria, 23(1), 99-105.

Fonseca, S. C. L., e Perez, S. C. J. G. A. (1999). Efeito de sais e da temperatura na germinação de sementes de olho-de-dragão (Anadenanthera pavonina L. FABACEAE). Revista Brasileira de Sementes, 21(2), 70-77.

Guedes, R. S., Alves, E. U., Oliveira, L. S. B., Andrade, L. A., Gonçalves, E. P., e Melo, P. A. R. F. (2011) Envelhecimento acelerado na avaliação da qualidade fisiológica de sementes de Dalbergia nigra (Vell.) Fr. All. Semina: Ciências Agrárias, 32(2), 443-450. DOI: 10.5433/1679-0359.2011v32n2p443

Lavezo, A., Braga, L. F., Batistão, A. C.; Bonfante, L. V. (2015). Estresse osmótico na germinação de sementes de Petiveria alliacea L. Revista Brasileira de Plantas Medicinais, 17(4), 622-630. DOI: 10.1590/1983-084X/14_026

Lemes, E. Q., Lopes, J. C., Nogueira, N. O., Silva, L. F., Gomes, D. e Pereira, D. S. Qualidade fisiológica de Cupania vernalis Cambess sob diferentes níveis de salinidade (2012). Revista Trópica - Ciencias Agrárias e Biologicas, 6(3), 147.

Lemes, E. Q. 2011. Qualidade Fisiológica de Sementes de Paineira em Função do Estádio de Maturação, Temperaturas Cardinais e Estresse Salino. Dissertação (Mestrado em Ciências Florestais), Universidade Federal do Espírito Santo, Centro de Ciências Agrárias.

Lima, B. G., e Torres, S. B. (2009). Estresses hídrico e salino na germinação de sementes de Zizyphus joazeiro Mart. (Rhamnaceae). Revista Caatinga, Mossoró, 22(4), 93-99.

Maguire, J. D. (1962). Speed of germination and in selection and evaluation for seedling emergence and vigour. Crop Science, 2, 176-177.

Matoso, S. C. G., e Salman, A. K. D. (2016). Fertilização orgânica em sistemas silvipastoris. Pesq. flor. bras., Colombo, 36(88), 519-526. DOI: 10.4336/2016.pfb.36.88.982

Mayer, A. M., e Poljakoff-Mayber, A. The germination of seeds. 4. ed. New York: Pergamon Press, 1989, 270 p.

Mendonça, A. V. R. (2006). Reabilitação de cavas de extração de argila e tolerância de espécies florestais à salinidade. Tese (Doutorado em Produção Vegetal) Universidade Federal do Norte Fluminense, Campos dos Goytacazes.

Mishra, A.; Sharma, S. D.; Khan, G. H. (2003) Improvement in physical and chemical properties of sodic soil by 3, 6 and 9 years old plantationsof Eucalyptus tereticornis: Biorejuvenation of sodic soil. Forest Ecology and Management, 184(1-3), 115-124.

Nakagawa, J. (1999). Testes de vigor baseados no desempenho das plântulas. In: Krzyzanowski, F.C.,

Vieira, R. D., França Neto, J. B. Vigor de Sementes: conceitos e testes. Londrina: Abrates, 21-24.

Nascimento, I. L. (2012). Superação da dormência em sementes de paineira-branca. Cerne, Lavras, 18(2), 285-291.

Pedrotti; A., Chagas, R. M., Ramos, V. C., Prata, A. P. N., Lucas, A. A. T., e Santos, P. B. (2015). Causas e consequências do processo de salinização dos solos. Revista Eletrônica em Gestão, Educação e Tecnologia Ambiental, Santa Maria, 19(2), 1308-1324. DOI: 105902/2236117016544

Pinto, I. O., Bastos, I. M. A. S., Erasmo, E. A. L., e Garcia, B. O. (2013). Efeitos do estresse salino sob sobre a porcentagem e indice de velocidade de germinação de sementes de Acacia mangium Willd. Palmas: UFT.

Ribeiro, M. C. C., Marques, B. M., e Amarro, J. Filho. (2001). Efeito da salinidade na germinação de sementes de quatro cultivares de girassol (Helianthus annuиs L.). Revista Brasileira de Sementes, Brasília, 23(1), 281-284.

Santos, C. A., Silva, N. V., Walter, L. S., Silva, E. C. A., e Nogueira, R. J. M. C. (2016). Germinação de sementes de duas espécies da caatinga sob déficit hídrico e salinidade. Pesq. flor. bras., Colombo, 36(87), 219- 224. DOI: 10.4336/2016.pfb.36.87.101

Silva, R. B. (2015). Ecofisiologia da germinação de sementes e produção de mudas de Parkia platycephala Benth. Tese (Doutorado em Ciências Florestais), Universidade Federal Rural de Pernambuco.

Silva, R. B. (2011). Ecofisiologia de Sementes de Piptadenia stipulacea (Benth.) Ducke. Dissertação (Mestrado em Ciências Florestais), Universidade Federal Rural de Pernambuco.

Souza, R. S. (2013). Potencial de espécies florestais nativas na fitoextração de sais. Dissertação (Mestrado em Ciências Florestais e Ambientais) Universidade Federal de Mato Grosso, Cuiabá.

SPSS Inc. (2001). Statistical Analysis Using SPSS. Chicago.

Stefanello, R., Garcia, D. C., Menezes, N. L., Muniz, M. F. B., e WRASSE, C. F. (2006). Efeito da luz, temperatura e estresse hídrico no potencial fisiológico de sementes de funcho. Revista Brasileira de Sementes, 28(2), 135-141.

Stefanello, R., Viana, B. B., e Neves, L. A. S. (2018). Germinação de sementes de Thymus vulgaris submetidas ao estresse salino. Caderno de Pesquisa, Santa Cruz do Sul, 30(2), 19-27. DOI: 10.17058/cp.v30i2.12220

Su, Y. H., North, H., Grignon, C., Thibaud, J. B., Sentenac, H., e Very, A. A. (2005). Regulation by external K+ in a maize inward shaker channel targets transport activity in the high concentration range. Plant Cell, 17(15), 32-48. 